\title{
Irreducible elements in multi-adjoint concept lattices*
}

\author{
Ma Eugenia Cornejo, ${ }^{1}$ Jesús Medina, ${ }^{2}$ Eloisa Ramírez ${ }^{1}$ \\ ${ }^{1}$ Departamento de Estadística e I.O. Universidad de Cádiz, Spain \\ Email: \{mariaeugenia.cornejo,eloisa.ramirez\}@uca.es \\ ${ }^{2}$ Departamento de Matemáticas. Universidad de Cádiz, Spain \\ Email: jesus.medina@uca.es
}

\begin{abstract}
One of the most important elements in a lattice are the irreducible elements. For example, when the lattice is finite, which is usual in the computational case, it forms a base from which the complete lattice is obtained.

These elements are also important in Formal Concept Analysis, since they are the basic information of a relational system.

In the general fuzzy framework of multi-adjoint concept lattices, this paper presents a characterization of the irreducible elements and so, a mechanism to detect the base information given in a general relational system. This result is applied to reduce the size of the concept lattices without losing and modifying important information.
\end{abstract}

Keywords: Formal concept analysis, fuzzy sets, irreducible element.

\section{Introduction}

Rudolf Wille introduced in the eighties [19] a mathematical environment to extract information from databases, which is called formal concept analysis (FCA).

The main idea is to detect in a considered database a set of attributes $A$, a set of objects $B$ and a relation between them $R \subseteq A \times B$, and use two operators to extract information (concept) and hierarchize it to obtain a complete lattice, which is called concept lattice.

FCA has become an important and appealing research topic from a theoretical perspective [18] as well as from an applied one [6, 7, 13, 14].

One important problem arises when the whole set of concepts needs to be computed, since the complexity is exponential, even more in the fuzzy case. Therefore, it is very interesting to provide strategies to reduce this complexity. In order to do so, one important procedure is to decrease the size of the concept lattice, trying to conserve the information given in the considered database.

\footnotetext{
* Partially supported by the Spanish Science Ministry projects TIN2009-14562-C05-03 and TIN2012-39353-C04-04, and by Junta de Andalucía project P09-FQM-5233.
}

There exist several mechanisms with this goal, however almost all of them modify the original concepts, such as the use of hedges [2] or, indeed, the original context, like granular computing [10], and others do not use fuzzy subsets of objects and attributes but, for instance, a crisp subset of objects and a fuzzy subset of attributes, as in [15].

Multi-adjoint concept lattices were introduced $[16,17]$ as a new general approach to formal concept analysis, in which the philosophy of the multi-adjoint paradigm was applied to the formal concept analysis, in order to provide a general framework that could conveniently accommodate different fuzzy approaches given in the literature, such as [1, 3, 8, 12]. Adjoint triples [5] are used as basic operators to carry out the calculus in this framework and so a general non-commutative environment, which allows different degrees of preference related to the set of objects and attributes, can easily be established.

The main result in this paper is the characterization of the $\wedge$-irreducible elements of a multi-adjoint concept lattice, using fuzzy subsets of attributes. As a consequence, we introduce a strategy to reduce the size of the multi-adjoint concept lattices, without modifying the original concepts and only considering the most representative ones. A similar development can be given to $\vee$-irreducible elements.

The organization of the paper is as follows: preliminary notions and results, together with the multi-adjoint concept lattice framework, are introduced in Section 2; the main result of this paper is given in Section 3, and Section 4 presents an application of this result to reduce the size of concept lattices. Lastly, the paper introduces several conclusions and pointers for further work.

\section{Preliminaries}

In order to make this paper as self-contained as possible, first of all, we recall the well-known definition of irreducible elements of a lattice.

Definition 1 Given a lattice $(L, \preceq)$, such that $\wedge, \vee$ are the meet and the join operators, and an element $x \in L$ verifying

\section{If $L$ has a top element $\top$, then $x \neq \top$.}


2. If $x=y \wedge z$, then $x=y$ or $x=z$, for all $y, z \in L$.

we call $x$ meet-irreducible ( $\wedge$-irreducible) element of L. Condition (2) is equivalent to

$2^{\prime}$. If $x<y$ and $x<z$, then $x<y \wedge z$, for all $y, z \in L$.

Hence, if $x$ is $\wedge$-irreducible, then it cannot be represented by the infimum of strictly greatest elements.

A join-irreducible ( $\mathrm{V}$-irreducible) element of $L$ is defined dually.

Another definition about lattice theory, which will be used later, is the following.

Definition 2 Let $(L, \preceq)$ be a lattice and $\varnothing \neq M \subseteq$ $L$. Then $(M, \preceq)$ is a sublattice of $(L, \preceq)$, if for each $a, b \in M$ we have that:

$$
\begin{aligned}
& a \vee b \in M, \text { and } \\
& a \wedge b \in M
\end{aligned}
$$

The main goal of this paper is to characterize the $\wedge$-irreducible elements of a fuzzy concept lattice framework. For that, we have considered the multi-adjoint concept lattice framework, since it is a general fuzzy setting which embeds other interesting frameworks and provides a great flexibility. Next, we recall this fuzzy concept lattice introduced in [17].

In the multi-adjoint concept lattice framework, the operators that we use to define the conceptforming operators are the adjoint triples, which are generalizations of a triangular norm (t-norm) and its residuated implication [9].

Definition 3 Let $\left(P_{1}, \leq_{1}\right),\left(P_{2}, \leq_{2}\right),\left(P_{3}, \leq_{3}\right)$ be posets and \&: $P_{1} \times P_{2} \rightarrow P_{3}, \swarrow: P_{3} \times P_{2} \rightarrow P_{1}$, $\nwarrow: P_{3} \times P_{1} \rightarrow P_{2}$ be mappings, then $(\&, \swarrow, \nwarrow)$ is an adjoint triple with respect to $P_{1}, P_{2}, P_{3}$ if:

$$
x \leq_{1} z \swarrow y \quad \text { iff } \quad x \& y \leq_{3} z \quad \text { iff } \quad y \leq_{2} z \nwarrow x
$$

where $x \in P_{1}, y \in P_{2}$ and $z \in P_{3}$.

Note that the commutativity property is not assumed for the conjunctor \&. As a consequence, there exist two different ways of generalising the well-known adjoint property between a t-norm and its residuated implication, depending on which argument is fixed. This is the reason why two implications are considered. Condition (1) is also called adjoint property.

A multi-adjoint frame is the basic structure which allows the existence of several adjoint triples with respect to $L_{1}, L_{2}, P$, where $\left(L_{1}, \preceq_{1}\right)$ and $\left(L_{2}, \preceq_{2}\right)$ are complete lattices. $L_{1}$ and $L_{2}$ are required to be lattices since the infimum on $L_{1}$ and $L_{2}$ are considered in the definition of the generalization of the concept-forming operators and a top element is needed. Moreover, the lattices $L_{1}$ and $L_{2}$ must be complete since the set of attributes $A$ and objects $B$, that will be considered, could be infinite.

Furthermore, considering different adjoint triples will add more flexibility to the language. For example, it was shown that they contribute to describe preference among objects or attributes.

Definition $4 A$ multi-adjoint frame $\mathcal{L}$ is a tuple

$\left(L_{1}, L_{2}, P, \preceq_{1}, \preceq_{2}, \leq, \&_{1}, \swarrow^{1}, \nwarrow_{1}, \ldots, \&_{n}, \swarrow^{n}, \nwarrow_{n}\right)$ where $\left(L_{1}, \preceq_{1}\right)$ and $\left(L_{2}, \preceq_{2}\right)$ are complete lattices, $(P, \leq)$ is a poset and, for all $i=1, \ldots, n,\left(\&_{i}, \swarrow^{i}\right.$ ,$\left.\nwarrow_{i}\right)$ is an adjoint triple with respect to $L_{1}, L_{2}, P$. Multi-adjoint frames are denoted as $\left(L_{1}, L_{2}, P, \&_{1}, \ldots, \&_{n}\right)$.

Given a frame, a multi-adjoint context is a tuple consisting of sets of objects, attributes and a fuzzy relation among them; in addition, the multi-adjoint approach also includes a function which assigns an adjoint triple to each pair of objects and attributes.

Definition 5 Let $\left(L_{1}, L_{2}, P, \&_{1}, \ldots, \&_{n}\right)$ be a multi-adjoint frame, a context is a tuple $(A, B, R, \sigma)$ such that $A$ and $B$ are non-empty sets (usually interpreted as attributes and objects, respectively), $R$ is a $P$-fuzzy relation $R: A \times B \rightarrow P$ and $\sigma: A \times B \rightarrow\{1, \ldots, n\}$ is a mapping which associates any element in $A \times B$ with some particular adjoint triple in the frame.

The denotation of $L_{2}^{B}$ and $L_{1}^{A}$ will be considered for the set of mappings $g: B \rightarrow L_{2}, f: A \rightarrow L_{1}$, respectively. On these sets a pointwise partial order can be considered from the partial orders in $\left(L_{1}, \preceq_{1}\right)$ and $\left(L_{2}, \preceq_{2}\right)$, which provides $L_{2}^{B}$ and $L_{1}^{A}$ with the structure of complete lattice, that is, $\left(L_{2}^{B}, \preceq_{2}\right)$ and ( $L_{1}^{A}, \preceq_{1}$ ) are complete lattices where $\preceq_{2}$ and $\preceq_{1}$ are defined pointwise, given $g_{1}, g_{2} \in L_{2}^{B}, f_{1}, f_{2} \in L_{1}^{A}$, $g_{1} \preceq_{2} g_{2}$ if and only if $g_{1}(b) \preceq_{2} g_{2}(b)$, for all $b \in B$; and $f_{1} \preceq_{1} f_{2}$ if and only if $f_{1}(a) \preceq_{1} f_{2}(a)$, for all $a \in A$.

Once we have fixed a multi-adjoint frame and a context for that frame, the concept-forming operators are denoted as ${ }^{\uparrow} \sigma: L_{2}^{B} \longrightarrow L_{1}^{A}$ and $\downarrow^{\sigma}: L_{1}^{A} \longrightarrow$ $L_{2}^{B}$ and are defined, for all $g \in L_{2}^{B}, f \in L_{1}^{A}$ and $a \in A, b \in B$, as

$$
\begin{aligned}
& g^{\uparrow_{\sigma}}(a)=\inf \left\{R(a, b) \swarrow^{\sigma(b)} g(b) \mid b \in B\right\} \\
& f^{\downarrow^{\sigma}}(b)=\inf \left\{R(a, b) \nwarrow_{\sigma(b)} f(a) \mid a \in A\right\}
\end{aligned}
$$

It is not difficult to show that these two arrows form a Galois connection [17]. In order to simplify the denotation we will write ${ }^{\uparrow}$ and $\downarrow$ instead of $\uparrow_{\sigma}$ and $\downarrow^{\sigma}$, respectively.

The notion of concept is defined as usual: a multiadjoint concept is a pair $\langle g, f\rangle$ satisfying that $g \in$ $L_{2}^{B}, f \in L_{1}^{A}$ and that $g^{\uparrow}=f$ and $f^{\downarrow}=g$; with $\left({ }^{\uparrow}, \downarrow\right)$ being the Galois connection defined above.

Given $g \in L_{2}^{B}$ (resp. $f \in L_{1}^{A}$ ), we will call the concept $\left\langle g^{\uparrow \downarrow}, g^{\uparrow}\right\rangle$ (resp. $\left\langle f^{\downarrow}, f^{\downarrow \uparrow}\right\rangle$ ) the closure concept of $g$ (resp. $f$ ). 
Finally, the definition of concept lattice in this framework is defined.

Definition 6 The multi-adjoint concept lattice associated with a multi-adjoint frame $\left(L_{1}, L_{2}, P, \&_{1}, \ldots, \&_{n}\right)$ and a context $(A, B, R, \sigma)$ is the set

$\mathcal{M}=\left\{\langle g, f\rangle \mid g \in L_{2}^{B}, f \in L_{1}^{A}\right.$ and $\left.g^{\uparrow}=f, f^{\downarrow}=g\right\}$

in which the ordering is defined by $\left\langle g_{1}, f_{1}\right\rangle \preceq$ $\left\langle g_{2}, f_{2}\right\rangle$ if and only if $g_{1} \preceq_{2} g_{2}$ (equivalently $f_{2} \preceq_{1}$ $\left.f_{1}\right)$.

In [17], the authors proved that the ordering just defined above provides $\mathcal{M}$ with the structure of complete lattice. Moreover, a representation theorem to multi-adjoint concept lattices was proven, which generalizes the classical one and different other fuzzy generalizations.

\section{The $\wedge$-irreducible elements of a multi-adjoint concept lattice}

This section characterizes the $\wedge$-irreducible elements of a multi-adjoint concept lattice. This fact is very important, since every concept is the infimum of $\wedge$-irreducible elements. Hence, an algorithm that builds lattices from the $\wedge$-irreducible elements will be applied to obtain the concept lattice.

Hereon, we will consider a multi-adjoint concept lattice $(\mathcal{M}, \preceq)$ associated with a multi-adjoint frame $\left(L_{1}, L_{2}, P, \&_{1}, \ldots, \&_{n}\right)$, a context $(A, B, R, \sigma)$, were $L_{1}, L_{2}, P, A$ and $B$, are finite, an index set $I$, such that $A=\left\{a_{i} \mid i \in I\right\}$, and the following specific family of fuzzy subsets of $L_{1}^{A}$.

Definition 7 For each $a_{i} \in A$, the fuzzy subsets of attributes $\phi_{i, x} \in L_{1}^{A}$ defined, for all $x \in L_{1}$, as

$$
\phi_{i, x}(a)= \begin{cases}x & \text { if } a=a_{i} \\ 0 & \text { if } a \neq a_{i}\end{cases}
$$

will be called fuzzy-attributes. The set of all fuzzyattributes will be denoted as $\Phi=\left\{\phi_{i, x} \mid a_{i} \in A, x \in\right.$ $\left.L_{1}\right\}$

Clearly, these mappings are generalizations of the crisp attributes and, moreover, they were also assumed in the proof of the representation theorem of multi-adjoint concept lattices.

The following result provides a first relation among a general fuzzy subset $f \in L_{1}^{A}$ and the fuzzyattributes $\phi_{i, x} \in L_{1}^{A}$.

Proposition 8 For all $f \in L_{1}^{A}$, we have that $f=$ $\bigvee_{i \in I} \phi_{i, f\left(a_{i}\right)}$.

Consequently, we obtain the following result.

Corollary 9 Given $f \in L_{1}^{A}$, we have that:

$$
f=\bigvee\left\{\phi_{i, x} \in \Phi \mid \phi_{i, x} \preceq_{1} f\right\}
$$

Therefore, each fuzzy subset of attributes $f$ can be written as the supremum of the fuzzy-attributes less than or equal to $f$. Hence, given $f \in L_{1}^{A}$, there exists a subset $\left\{\phi_{j, x_{j}} \mid j \in J\right\} \subseteq \Phi$, such that $f=$ $\bigvee_{j \in J} \phi_{j, x_{j}}$.

Note that it is possible that there exist $j, k \in J$ and $i \in I$, such that $\phi_{j, x_{j}}=\phi_{i, x}$ and $\phi_{j, x_{j}}=\phi_{i, x^{\prime}}$, with $x, x^{\prime} \in L_{1}$ and $x \neq x^{\prime}$.

The following result characterizes the $\wedge$ irreducible elements of a multi-adjoint concept lattice.

Theorem 10 The set of $\wedge$-irreducible elements of $\mathcal{M}, M_{F}(A, B, R, \sigma)$, is formed by the pairs $\left\langle\phi_{i, x}^{\downarrow}, \phi_{i, x}^{\downarrow \uparrow}\right\rangle$ in $\mathcal{M}$, with $a_{i} \in A$ and $x \in L_{1}$, such that

$$
\phi_{i, x}^{\downarrow} \neq \bigwedge\left\{\phi_{j, x_{j}}^{\downarrow} \mid \phi_{j, x_{j}} \in \Phi, \phi_{i, x}^{\downarrow} \prec_{2} \phi_{j, x_{j}}^{\downarrow}\right\}
$$

and $\phi_{i, x}^{\downarrow} \neq g_{\top}$, where $\top$ is the maximum element in $L_{2}$ and $g_{\top}: B \rightarrow L_{2}$ is the fuzzy subset defined as $g_{\top}(b)=\top$, for all $b \in B$.

Note that, in this result, the expression $\phi_{i, x}^{\downarrow} \neq$ $\bigwedge\left\{\phi_{j, x_{j}}^{\downarrow} \mid \phi_{i, x}^{\downarrow} \prec_{2} \phi_{j, x_{j}}^{\downarrow}\right\}$ may be replaced by $\phi_{i, x}^{\downarrow} \prec_{2}$ $\bigwedge\left\{\phi_{j, x_{j}}^{\downarrow} \mid \phi_{i, x}^{\downarrow} \prec_{2} \phi_{j, x_{j}}^{\downarrow}\right\}$ since both are equivalent. However, we prefer to use the inequality in order to emphasize the difference.

From the previous theorem we obtain that all the $\wedge$-irreducible elements are the closure concept of fuzzy-attributes and no more concepts can be $\wedge$-irreducible elements. Moreover, in order to obtain these elements, we only need to check if $\phi_{i, x}^{\downarrow}$ is the infimum of the fuzzy-attributes that are greater than $\phi_{i, x}^{\downarrow}$, and we do not need to consider the whole set of fuzzy subsets of attributes that are greater.

Now, we present an example in which we ascertain Theorem 10 in order to obtain the fuzzy $\wedge$ irreducible elements of a particular multi-adjoint concept lattice.

Example 11 In this example we consider $L=$ $\{0.0,0.5,1.0\}, \& G$ the Gödel conjunctor defined on $L,\left(L, \preceq, \&_{G}\right)$ the multi-adjoint frame and the context $(A, B, R, \sigma)$, where $A=\left\{a_{1}, a_{2}, a_{3}, a_{4}, a_{5}, a_{6}\right\}$, $B=\left\{b_{1}, b_{2}, b_{3}\right\}, R: A \times B \rightarrow L$ is given by Table 1 , and $\sigma$ is constant.

Table 1: Relation $R$ of Example 11.

\begin{tabular}{|c||c|c|c|}
\hline$R$ & $b_{1}$ & $b_{2}$ & $b_{3}$ \\
\hline \hline$a_{1}$ & 0.5 & 1.0 & 0.0 \\
\hline$a_{2}$ & 0.5 & 1.0 & 0.0 \\
\hline$a_{3}$ & 0.5 & 0.5 & 0.5 \\
\hline$a_{4}$ & 1.0 & 1.0 & 0.5 \\
\hline$a_{5}$ & 1.0 & 0.5 & 1.0 \\
\hline$a_{6}$ & 1.0 & 0.5 & 0.5 \\
\hline
\end{tabular}

The concept lattice $(\mathcal{M}, \preceq)$, associated with the considered frame and context, has 9 concepts listed 
below.

$$
\begin{aligned}
C_{0}= & \left\langle\left\{0.5 / b_{1}, 0.5 / b_{2}\right\},\right. \\
& \left.\left\{1.0 / a_{1}, 1.0 / a_{2}, 1.0 / a_{3}, 1.0 / a_{4}, 1.0 / a_{5}, 1.0 / a_{6}\right\}\right\rangle \\
C_{1}= & \left\langle\left\{0.5 / b_{1}, 1.0 / b_{2}\right\},\right. \\
& \left.\left\{1.0 / a_{1}, 1.0 / a_{2}, 0.5 / a_{3}, 1.0 / a_{4}, 0.5 / a_{5}, 0.5 / a_{6}\right\}\right\rangle \\
C_{2}= & \left\langle\left\{1.0 / b_{1}, 0.5 / b_{2}\right\},\right. \\
& \left.\left\{1.0 / a_{1}, 0.5 / a_{2}, 0.5 / a_{3}, 1.0 / a_{4}, 1.0 / a_{5}, 1.0 / a_{6}\right\}\right\rangle \\
C_{3}= & \left\langle\left\{0.5 / b_{1}, 0.5 / b_{2}, 0.5 / b_{3}\right\},\right. \\
& \left.\left\{1.0 / a_{3}, 1.0 / a_{4}, 1.0 / a_{5}, 1.0 / a_{6}\right\}\right\rangle \\
C_{4}= & \left\langle\left\{1.0 / b_{1}, 1.0 / b_{2}\right\},\right. \\
& \left.\left\{1.0 / a_{1}, 0.5 / a_{2}, 0.5 / a_{3}, 1.0 / a_{4}, 0.5 / a_{5}, 0.5 / a_{6}\right\}\right\rangle \\
C_{5}= & \left\langle\left\{1.0 / b_{1}, 0.5 / b_{2}, 0.5 / b_{3}\right\},\right. \\
& \left.\left\{0.5 / a_{3}, 1.0 / a_{4}, 1.0 / a_{5}, 1.0 / a_{6}\right\}\right\rangle \\
C_{6}= & \left\langle\left\{1.0 / b_{1}, 1.0 / b_{2}, 0.5 / b_{3}\right\},\right. \\
& \left.\left\{0.5 / a_{3}, 1.0 / a_{4}, 0.5 / a_{5}, 0.5 / a_{6}\right\}\right\rangle \\
C_{7}= & \left\langle\left\{1.0 / b_{1}, 0.5 / b_{2}, 1.0 / b_{3}\right\},\right. \\
& \left.\left\{0.5 / a_{3}, 0.5 / a_{4}, 1.0 / a_{5}, 0.5 / a_{6}\right\}\right\rangle \\
C_{8}= & \left\langle\left\{1.0 / b_{1}, 1.0 / b_{2}, 1.0 / b_{3}\right\},\right. \\
& \left.\left\{0.5 / a_{3}, 0.5 / a_{4}, 0.5 / a_{5}, 0.5 / a_{6}\right\}\right\rangle
\end{aligned}
$$

The Hasse diagram of this lattice is shown in Figure 1.

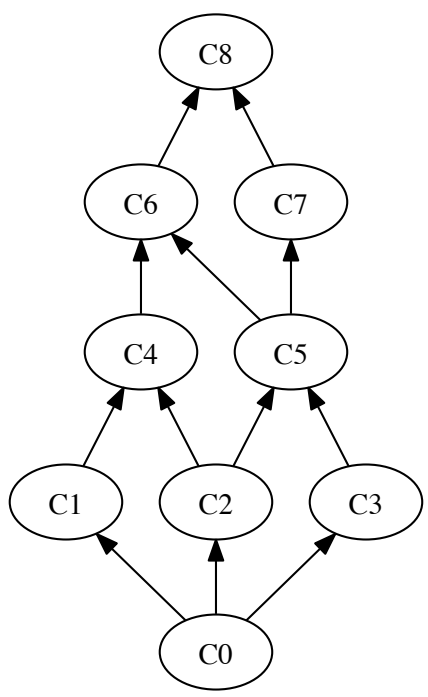

Figure 1: The Hasse diagram of $(\mathcal{M}, \preceq)$

With respect to the fuzzy-attributes, clearly, $\left\langle\phi_{a, 0.0}^{\downarrow}, \phi_{a, 0.0}^{\downarrow \uparrow}\right\rangle=C_{8}$, for all $a \in A$. Moreover, $\left\langle\phi_{3,0.5}^{\downarrow}, \phi_{3,0.5}^{\downarrow \uparrow}\right\rangle,\left\langle\phi_{4,0.5}^{\downarrow}, \phi_{4,0.5}^{\downarrow \uparrow}\right\rangle$ and $\left\langle\phi_{5,0.5}^{\downarrow}, \phi_{5,0.5}^{\downarrow \uparrow}\right\rangle$ are $C_{8}$. The rest are

$$
\begin{aligned}
\left\langle\phi_{1,0.5}^{\downarrow}, \phi_{1,0.5}^{\downarrow \uparrow}\right\rangle= & \left\langle\phi_{2,0.5}^{\downarrow}, \phi_{2,0.5}^{\downarrow \uparrow}\right\rangle=C_{4} \\
\left\langle\phi_{1,1.0}^{\downarrow}, \phi_{1,1.0}^{\downarrow \uparrow}\right\rangle= & \left\langle\phi_{2,1.0}^{\downarrow}, \phi_{2,1.0}^{\downarrow \uparrow}\right\rangle=C_{1} \\
& \left\langle\phi_{3,1.0}^{\downarrow}, \phi_{3,1.0}^{\downarrow \uparrow}\right\rangle=C_{3} \\
& \left\langle\phi_{4,1.0}^{\downarrow}, \phi_{4,1.0}^{\downarrow \uparrow}\right\rangle=C_{6} \\
& \left\langle\phi_{5,1.0}^{\downarrow}, \phi_{5,1.0}^{\downarrow \uparrow}\right\rangle=C_{7} \\
& \left\langle\phi_{6,1.0}^{\downarrow}, \phi_{6,1.0}^{\downarrow \uparrow}\right\rangle=C_{5}
\end{aligned}
$$

Obtaining the concepts associated with the fuzzyattributes in $\Phi$, which are $C_{1}, C_{3}, C_{4}, C_{6}, C_{7}$ and $C_{8}$. Referring to the Hasse diagram, it is not hard to see that the set of $\wedge$-irreducible elements of $\mathcal{M}$ is

$$
M_{F}(A, B, R, \sigma)=\left\{C_{1}, C_{3}, C_{4}, C_{6}, C_{7}\right\}
$$

Moreover, these irreducible concepts verify the conditions of the previous theorem, namely, they are concepts $\left\langle\phi_{i, x}^{\downarrow}, \phi_{i, x}^{\downarrow \uparrow}\right\rangle$ associated with fuzzyattributes satisfying $\phi_{i, x}^{\downarrow} \neq \bigwedge\left\{\phi_{j, x_{j}}^{\downarrow} \mid \phi_{i, x}^{\downarrow} \prec_{2}\right.$ $\left.\phi_{j, x_{j}}^{\downarrow}\right\}$ and $\phi_{i, x}^{\downarrow} \neq g_{\top}$. For instance, $\phi_{6,1.0}^{\downarrow}$ is equal to the infimum of $\phi_{4,1.0}^{\downarrow}$ and $\phi_{5,1.0}^{\downarrow}$, and it is strictly less than these fuzzy subsets. Hence, the concept generated by $\phi_{6,1.0}^{\downarrow}$ is not a meet irreducible element and so, $\left\langle\phi_{6,1.0}^{\downarrow}, \phi_{6,1.0}^{\downarrow \uparrow}\right\rangle=C_{5}$ is not in $M_{F}(A, B, R, \sigma)$.

If we now consider $C_{3}=\left\langle\phi_{3,1.0}^{\downarrow}, \phi_{3,1.0}^{\downarrow \uparrow}\right\rangle$ and the computations, it is not possible to express this element as infimum of elements $\phi_{j, x_{j}}^{\downarrow}$, such that $\phi_{3,1.0}^{\downarrow} \prec_{2} \phi_{j, x_{j}}^{\downarrow}$, and, moreover, $\phi_{3,1.0}^{\downarrow}$ is not the topelement. A similar deduction can be given for the rest of concepts generated from a fuzzy-attribute, obtaining the set of $\wedge$-irreducible elements of $(\mathcal{M}, \preceq)$ from Theorem 10.

From the viewpoint of efficiency, it would be great if each attribute was associated with only one $\wedge$ irreducible element in $M_{F}(A, B, R, \sigma)$. However, this is not true in general, for instance, the last example provides an attribute that is associated with two $\wedge$-irreducible elements. We have that the concepts $C_{4}=\left\langle\phi_{2,0.5}^{\downarrow}, \phi_{2,0.5}^{\downarrow \uparrow}\right\rangle$ and $C_{1}=\left\langle\phi_{2,1.0}^{\downarrow}, \phi_{2,1.0}^{\downarrow \uparrow}\right\rangle$ are different $\wedge$-irreducible elements of $\mathcal{M}$. Therefore, two different $\wedge$-irreducible concepts can be $\left\langle\phi_{i, x}^{\downarrow}, \phi_{i, x}^{\downarrow \uparrow}\right\rangle$ and $\left\langle\phi_{i, x}^{\downarrow}, \phi_{i, x^{\prime}}^{\downarrow \uparrow}\right\rangle$, satisfying that $x, x^{\prime} \in L$ and $x \neq x^{\prime}$.

This is the main reason why we cannot write the elements of $\Phi$ as $\phi_{i, x_{i}}$, with $i \in I$, that is, only one $x_{i}$ associated with one attribute $a_{i}$.

Theorem 10 also provides a mechanism to obtain the multi-adjoint concept lattice related to a context. Since it characterizes the $\wedge$-irreducible elements by the closure concepts of fuzzy-attributes, which satisfy a specific property, we can only consider these fuzzy-attributes in order to build the concept lattice. This is the basic idea used in Algorithm 1.

Note that in Line 10 any procedure to obtain a lattice from the irreducible elements can be considered.

\section{Application to reduce the size of multi-adjoint concept lattices}

One of the most recognized problems in FCA is to reduce the size of the concept lattices $[2,10,11,15]$. However, several of the existing mechanisms modify the information given by the concepts. This section uses the previous characterization in order to provide a new procedure to reduce the size of the multiadjoint concept lattices, without modifying the information given by the context, but beginning from the fuzzy-attributes that really can represent an attribute. 
input : $A=\left\{a_{1}, \ldots, a_{n}\right\}, B, R, \sigma, L_{1}=$ $\left\{\perp, x_{1}, \ldots, x_{m}\right\}, L_{2}, P$

output: Meet-irred, MAConceptLattice

$1 D=\varnothing$

2 for $i \leftarrow 1$ to $n$ and $x \leftarrow 1$ to $m$ do

$3 \quad$ Compute $\phi_{i, x}^{\downarrow}$

$4 \quad$ if $\phi_{i, x}^{\downarrow} \notin D$ then

$5 \quad \mid$ add $\phi_{i, x}^{\downarrow}$ to $D$

6 end

7 end

8 Compute $I=\left\{\phi_{i, x}^{\downarrow} \in D \mid \phi_{i, x}^{\downarrow}=\right.$ $\bigwedge \phi_{j, x_{j}}^{\downarrow}$ such that $\left.\phi_{j, x_{j}}^{\downarrow} \in D, \phi_{i, x}^{\downarrow} \prec_{2} \phi_{j, x_{j}}^{\downarrow}\right\}$;

9 Meet-irred $=D \backslash I$;

10 Build the concept lattice from Meet-irred;

Algorithm 1: Obtaining a multi-adjoint concept lattice

From Theorem 10 we have that every fuzzyattribute $\phi_{i, x}$ associated with an attribute $a_{i}$, with $x \in L$, can be considered in the computation of the concept lattice, if this fuzzy-attribute generates a meet irreducible element. For instance, if $L=\{0.0,0.2,0.5,0.7,1.0\}$, then $\phi_{i, 0.2}$ could be considered, when the value for the attribute $a_{i}$ is not representative, since $\alpha=0.2$ is very small. Therefore, it could be more interesting to consider the fuzzy-attributes $\phi_{i, x}$ in which the value $x$ exceeds a threshold $\alpha$.

From the irreducible elements of $M_{F}(A, B, R, \sigma)$ we will only consider the fuzzy-attributes with a considerable value. Hence, given a threshold $\alpha$, we will only assume the fuzzy-attributes of each attribute $a_{i}$ that provides $a_{i}$ with a value greater than $\alpha$, that is, we consider the following set of meet irreducible elements of $(\mathcal{M}, \preceq)$ :

$M_{F}(A)_{\alpha}=\left\{\left\langle\phi_{i, x}^{\downarrow}, \phi_{i, x}^{\downarrow \uparrow}\right\rangle \in M_{F}(A, B, R, \sigma) \mid \alpha \preceq_{1} x\right\}$

Hence, we only consider the concepts of $(\mathcal{M}, \preceq)$, which are obtained from the infimum of elements of $M_{F}(A)_{\alpha}$. Moreover, in order to obtain a complete lattice we also need to consider the greatest element in $(\mathcal{M}, \preceq)$, that is $\left\langle g_{\top}, g_{\top}^{\uparrow}\right\rangle$.

Definition 12 Given $\alpha \in L_{1}$, the set $\mathcal{M}_{\alpha}$, defined as:

$\left\{\langle g, f\rangle \in \mathcal{M} \mid g=\bigwedge_{j \in J} \phi_{j, x_{j}}^{\downarrow}\right.$, with $\left.\phi_{j, x_{j}} \in M_{F}(A)_{\alpha}\right\}$

together with $\left\{\left\langle g_{\top}, g_{\top}^{\uparrow}\right\rangle\right\}$, is called irreducible $\alpha$-cut of $\mathcal{M}$.

This set, with the ordering defined in $\mathcal{M}$, restricted to $\mathcal{M}_{\alpha}$, forms a lattice.

Theorem 13 For each $\alpha \in L_{1}$, if $\mathcal{M}_{\alpha}$ is an irreducible $\alpha$-cut of $\mathcal{M}$, then $\left(\mathcal{M}_{\alpha}, \preceq\right)$ is a sublattice of $(\mathcal{M}, \preceq)$.
Therefore, $\left(\mathcal{M}_{\alpha}, \preceq\right)$ is a concept lattice and, consequently, the following result holds.

Corollary 14 Given $(\mathcal{M}, \preceq)$ and $\left(\mathcal{M}_{\alpha}, \preceq\right)$, we have that

$$
\begin{aligned}
\operatorname{Ext}\left(\mathcal{M}_{\alpha}\right) & \subseteq \operatorname{Ext}(\mathcal{M}) \\
\operatorname{Int}\left(\mathcal{M}_{\alpha}\right) & \subseteq \operatorname{Int}(\mathcal{M})
\end{aligned}
$$

where $\operatorname{Ext}\left(\mathcal{M}_{\alpha}\right), \operatorname{Ext}(\mathcal{M}), \operatorname{Int}\left(\mathcal{M}_{\alpha}\right)$ and $\operatorname{Int}(\mathcal{M})$ are the extension and intension sets of the concept lattices $\mathcal{M}_{\alpha}$ and $\mathcal{M}$, respectively.

A similar procedure can be developed with respect to the join irreducible elements.

The granularity of the carriers considered in Example 11 is very limited, the lattice $L=$ $\{0.0,0.5,1.0\}$ has only three values and therefore, the unique value $\alpha$ that could introduce some reduction is $\alpha=1.0$. In this case, $\phi_{1,0.5}$ and $\phi_{2,0.5}$ are not considered to obtain the concepts of $\mathcal{M}_{\alpha}$ and, for that reason, the meet irreducible element $C_{4}$ is not obtained by another fuzzy attribute. Thus,

$$
M_{F}(A)_{1.0}=\left\{C_{1}, C_{3}, C_{6}, C_{7}\right\}
$$

The irreducible 1.0-cut of $\mathcal{M}$ is given in Figure 2, in which $C_{4}$ and $C_{2}$ do not appear. $C_{4}$ has been erased by the threshold $\alpha$, and $C_{2}$ is not present because it is only obtained by the infimum of $C_{4}$ and $C_{5}$.

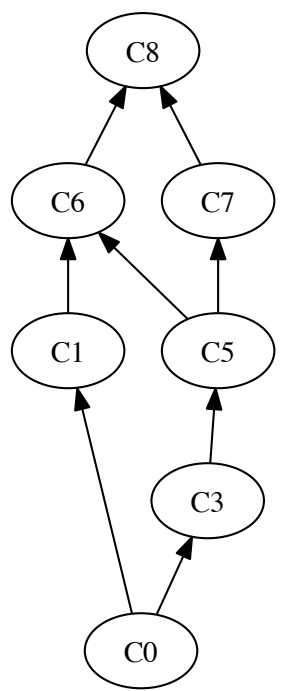

Figure 2: The Hasse diagram of $\left(\mathcal{M}_{1.0}, \preceq\right)$

Next, we will present another more general example which shows different irreducible $\alpha$-cuts of a multi-adjoint concept lattice.

Example 15 Given the frame $\left(L, \preceq, \&_{G}\right)$, where $L=\{0.0,0.2,0.4,0.6,0.8,1.0\}$ and $\&_{G}$ is the Gödel conjunctor, the context $(A, B, R, \sigma)$, where $A=$ $\left\{a_{1}, a_{2}, a_{3}\right\}, B=\left\{b_{1}, b_{2}, b_{3}\right\}, R: A \times B \rightarrow L$ is defined from Table 2, and $\sigma$ is constant, the concept lattice $(\mathcal{M}, \preceq)$ has 14 concepts: 
Table 2: Relation $R$ of Example 15.

\begin{tabular}{|c||c|c|c|}
\hline$R$ & $b_{1}$ & $b_{2}$ & $b_{3}$ \\
\hline \hline$a_{1}$ & 0.2 & 0.8 & 0.4 \\
\hline$a_{2}$ & 0.4 & 1.0 & 0.8 \\
\hline$a_{3}$ & 0.6 & 0.0 & 1.0 \\
\hline
\end{tabular}

$C_{0}=\left\langle\left\{0.2 / b_{1}, 0.4 / b_{3}\right\},\left\{1.0 / a_{1}, 1.0 / a_{2}, 1.0 / a_{3}\right\}\right\rangle$

$C_{1}=\left\langle\left\{0.2 / b_{1}, 0.8 / b_{2}, 0.4 / b_{3}\right\},\left\{1.0 / a_{1}, 1.0 / a_{2}\right\}\right\rangle$

$C_{2}=\left\langle\left\{0.2 / b_{1}, 0.8 / b_{3}\right\},\left\{0.4 / a_{1}, 1.0 / a_{2}, 1.0 / a_{3}\right\}\right\rangle$

$C_{3}=\left\langle\left\{0.2 / b_{1}, 1.0 / b_{2}, 0.4 / b_{3}\right\},\left\{0.8 / a_{1}, 1.0 / a_{2}\right\}\right\rangle$

$C_{4}=\left\langle\left\{0.2 / b_{1}, 1.0 / b_{3}\right\},\left\{0.4 / a_{1}, 0.8 / a_{2}, 1.0 / a_{3}\right\}\right\rangle$

$C_{5}=\left\langle\left\{0.4 / b_{1}, 0.8 / b_{3}\right\},\left\{0.2 / a_{1}, 1.0 / a_{2}, 1.0 / a_{3}\right\}\right\rangle$

$C_{6}=\left\langle\left\{0.2 / b_{1}, 1.0 / b_{2}, 0.8 / b_{3}\right\},\left\{0.4 / a_{1}, 1.0 / a_{2}\right\}\right\rangle$

$C_{7}=\left\langle\left\{0.4 / b_{1}, 1.0 / b_{3}\right\},\left\{0.2 / a_{1}, 0.8 / a_{2}, 1.0 / a_{3}\right\}\right\rangle$

$C_{8}=\left\langle\left\{0.2 / b_{1}, 1.0 / b_{2}, 1.0 / b_{3}\right\},\left\{0.4 / a_{1}, 0.8 / a_{2}\right\}\right\rangle$

$C_{9}=\left\langle\left\{0.4 / b_{1}, 1.0 / b_{2}, 0.8 / b_{3}\right\},\left\{0.2 / a_{1}, 1.0 / a_{2}\right\}\right\rangle$

$C_{10}=\left\langle\left\{0.6 / b_{1}, 1.0 / b_{3}\right\},\left\{0.2 / a_{1}, 0.4 / a_{2}, 1.0 / a_{3}\right\}\right\rangle$

$C_{11}=\left\langle\left\{0.4 / b_{1}, 1.0 / b_{2}, 1.0 / b_{3}\right\},\left\{0.2 / a_{1}, 0.8 / a_{2}\right\}\right\rangle$

$C_{12}=\left\langle\left\{1.0 / b_{1}, 1.0 / b_{3}\right\},\left\{0.2 / y_{1}, 0.4 / a_{2}, 0.6 / a_{3}\right\}\right\rangle$

$C_{13}=\left\langle\left\{1.0 / b_{1}, 1.0 / b_{2}, 1.0 / b_{3}\right\},\left\{0.2 / a_{1}, 0.4 / a_{2}\right\}\right\rangle$

and they are hierarchized as Figure 3 shows.

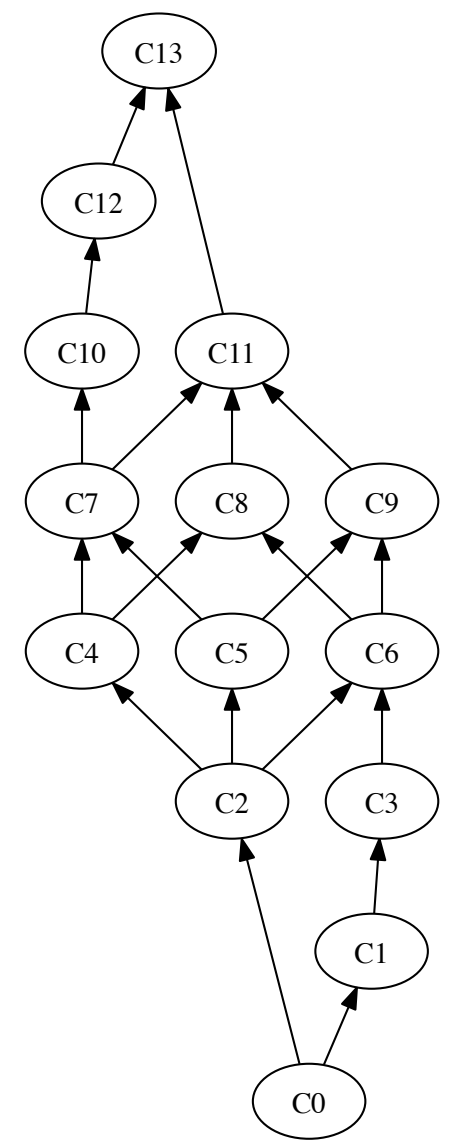

Figure 3: The Hasse diagram of $(\mathcal{M}, \preceq)$

With respect to the fuzzy-attributes, we have that $\left\langle\phi_{a, 0.0}^{\downarrow}, \phi_{a, 0.0}^{\downarrow \uparrow}\right\rangle=C_{13}$, for all $a \in A$. Moreover, $\left\langle\phi_{1,0.2}^{\downarrow}, \phi_{1,0.2}^{\downarrow \uparrow}\right\rangle,\left\langle\phi_{2,0.2}^{\downarrow}, \phi_{2,0.2}^{\downarrow \uparrow}\right\rangle$ and $\left\langle\phi_{2,0.4}^{\downarrow}, \phi_{2,0.4}^{\downarrow \uparrow}\right\rangle$ are also the maximum concept $C_{13}$.
The other fuzzy-attributes generate the next concepts:

$$
\begin{aligned}
&\left\langle\phi_{1,0.4}^{\downarrow}, \phi_{1,0.4}^{\downarrow \uparrow}\right\rangle=C_{8} \\
&\left\langle\phi_{1,0.6}^{\downarrow}, \phi_{1,0.6}^{\downarrow \uparrow}\right\rangle=\left\langle\phi_{1,0.8}^{\downarrow}, \phi_{1,0.8}^{\downarrow \uparrow}\right\rangle= C_{3} \\
&\left\langle\phi_{1,1.0}^{\downarrow}, \phi_{1,1.0}^{\downarrow \uparrow}\right\rangle=C_{1} \\
&\left\langle\phi_{2,0.6}^{\downarrow}, \phi_{2,0.6}^{\downarrow \uparrow}\right\rangle=\left\langle\phi_{2,1.0}^{\downarrow}, \phi_{2,1.0}^{\downarrow \uparrow}\right\rangle=C_{9}\left\langle\phi_{2,0.8}^{\downarrow}, \phi_{2,0.8}^{\downarrow \uparrow}\right\rangle=C_{11} \\
&\left\langle\phi_{3,0.2}^{\downarrow}, \phi_{3,0.2}^{\downarrow \uparrow}\right\rangle=\left\langle\phi_{3,0.4}^{\downarrow}, \phi_{3,0.4}^{\downarrow \uparrow}\right\rangle \\
&=\left\langle\phi_{3,0.6}^{\downarrow}, \phi_{3,0.6}^{\downarrow \uparrow}\right\rangle=C_{12} \\
&\left\langle\phi_{3,0.8}^{\downarrow}, \phi_{3,0.8}^{\downarrow \uparrow}\right\rangle=\left\langle\phi_{3,1.0}^{\downarrow}, \phi_{3,1.0}^{\downarrow \uparrow}\right\rangle=C_{10}
\end{aligned}
$$

From Theorem 10 and Figure 3, the $\wedge$-irreducible elements of $\mathcal{M}$ are:

$$
M_{F}(A, B, R, \sigma)=\left\{C_{1}, C_{3}, C_{8}, C_{9}, C_{10}, C_{11}, C_{12}\right\}
$$

Now, we consider a pair of examples of irreducible $\alpha$-cuts concept lattices of $\mathcal{M}$. In order to obtain proper concept lattices we will assume $\alpha=0.8$ and $\alpha=1.0$.

The first one considers the fuzzy-attributes $\phi_{i, x} \in$ $\Phi$ which generate a meet irreducible element of $\mathcal{M}$, satisfying that $\alpha \leq x$. Therefore, the set of meet irreducible elements considered to build the concept lattice $\mathcal{M}_{0.8}$ is:

$$
\left\{\phi_{1,0.8}^{\downarrow}, \phi_{1,1.0}^{\downarrow}, \phi_{2,0.8}^{\downarrow}, \phi_{2,1.0}^{\downarrow}, \phi_{3,1.0}^{\downarrow}\right\}
$$

Note that $\phi_{3,0.8}^{\downarrow}$ is really considered, since it is equal to $\phi_{3,1.0}^{\downarrow}$, which is already in the previous set.

Consequently, the concept lattice obtained is shown in Figure 4.

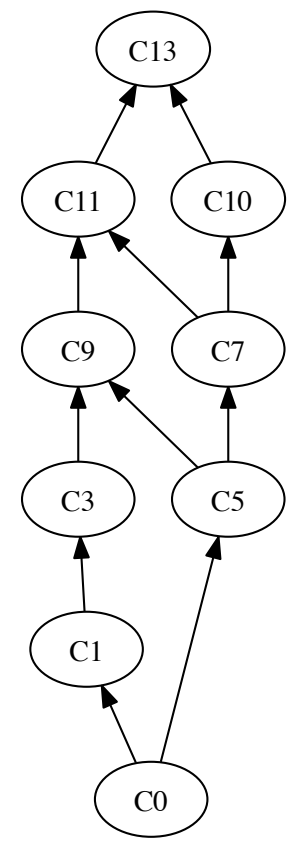

Figure 4: Concept lattice $\left(\mathcal{M}_{0.8}, \preceq\right)$

The second considered concept lattice is $\mathcal{M}_{1.0}$. In this case, the elements that we assume to build the irreducible 1.0-cut are:

$$
\left\{\phi_{1,1.0}^{\downarrow}, \phi_{2,1.0}^{\downarrow}, \phi_{3,1.0}^{\downarrow}\right\}
$$


and the obtained complete lattice is given in Figure 5 .

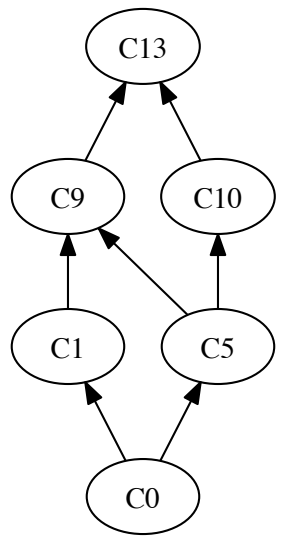

Figure 5: Concept lattice $\left(\mathcal{M}_{1.0}, \preceq\right)$

\section{Conclusions and future work}

A characterization of the $\wedge$-irreducible elements of a multi-adjoint concept lattice has been introduced using fuzzy-attributes. These elements are very important, since from them the whole concept lattice is built. From this characterization, we have introduced a strategy to reduce the size of the multiadjoint concept lattices, with the advantage that the subset of meet irreducible elements build a sublattice of the original concept lattice, and so, the original information given by the concepts is neither altered nor modified and the most representative knowledge is conserved.

In the future, we will compare the introduced size reduction method with the existing ones, and, using Theorem 10, a classification in the set of attributes will be studied and its applicability in the attribute implications framework [4].

\section{References}

[1] R. Bělohlávek. Concept lattices and order in fuzzy logic. Annals of Pure and Applied Logic, 128:277-298, 2004.

[2] R. Belohlavek and V. Vychodil. Reducing the size of fuzzy concept lattices by hedges. In The 2005 IEEE International Conference on Fuzzy Systems, pages 663-668, 2005.

[3] A. Burusco and R. Fuentes-González. Construction of the $L$-fuzzy concept lattice. Fuzzy Sets and Systems, 97(1):109-114, 1998.

[4] R. Bělohlávek and V. Vychodil. Attribute implications in a fuzzy setting. In Proceedings of the 4 th international conference on Formal Concept Analysis, ICFCA'06, pages 45-60, Berlin, Heidelberg, 2006. Springer-Verlag.

[5] M. Cornejo, J. Medina, and E. Ramírez. A comparative study of adjoint triples. Fuzzy Sets and Systems, 211:1-14, 2012.
[6] A. Formica. Concept similarity in fuzzy formal concept analysis for semantic web. International Journal of Uncertainty, Fuzziness and Knowlege-Based Systems, 18(2):153-167, 2010.

[7] B. Galitsky and J. D. L. Rosa. Concept-based learning of human behavior for customer relationship management. Information Sciences, 181(10):2016-2035, 2011.

[8] G. Georgescu and A. Popescu. Noncommutative fuzzy Galois connections. Soft Computing, 7(7):458-467, 2003.

[9] P. Hájek. Metamathematics of Fuzzy Logic. Trends in Logic. Kluwer Academic, 1998.

[10] X. Kang, D. Li, S. Wang, and K. Qu. Formal concept analysis based on fuzzy granularity base for different granulations. Fuzzy Sets and Systems, 203(0):33 - 48, 2012.

[11] J. Konecny, J. Medina, and M. O. Aciego. Intensifying hedges and the size of multi-adjoint concept lattices with heterogeneous conjunctors. In The 9th International Conference on Concept Lattices and Their Applications, pages 245-256, 2012.

[12] S. Krajči. A generalized concept lattice. Logic Journal of IGPL, 13(5):543-550, 2005.

[13] C. Kumar and S. Srinivas. Mining associations in health care data using formal concept analysis and singular value decomposition. Journal of Biological Systems, 18(4):787-807, 2010.

[14] B. Li, X. Sun, and H. Leung. Combining concept lattice with call graph for impact analysis. Advances in Engineering Software, 53(0):1 $-13,2012$.

[15] L. Li and J. Zhang. Attribute reduction in fuzzy concept lattices based on the t-implication. Knowledge-Based Systems, 23(6):497 - 503, 2010.

[16] J. Medina and M. Ojeda-Aciego. Multiadjoint t-concept lattices. Information Sciences, 180(5):712-725, 2010.

[17] J. Medina, M. Ojeda-Aciego, and J. RuizCalviño. Formal concept analysis via multiadjoint concept lattices. Fuzzy Sets and Systems, 160(2):130-144, 2009.

[18] K.-S. Qu and Y.-H. Zhai. Generating complete set of implications for formal contexts. Knowledge-Based Systems, 21:429-433, 2008.

[19] R. Wille. Restructuring lattice theory: an approach based on hierarchies of concepts. In I. Rival, editor, Ordered Sets, pages 445-470. Reidel, 1982. 